\title{
Early stroke-related deep venous thrombosis: risk factors and influence on outcome
}

\author{
Jan Bembenek • Michal Karlinski - Adam Kobayashi • \\ Anna Czlonkowska
}

Published online: 26 February 2011

(c) The Author(s) 2011. This article is published with open access at Springerlink.com

\begin{abstract}
Deep venous thrombosis (DVT) is a serious complication of various medical conditions including acute stroke. Our aim was to identify the occurrence of early stroke-related DVT, risk factors for its development and the influence on outcome. The study involved consecutive patients admitted to our center due to acute ischaemic $(n=$ $278)$ or haemorrhagic $(n=12)$ stroke during a 16-month period. We collected data on their pre-stroke health status, neurological deficit on admission and baseline serum CRP and fibrinogen level. Ultrasonographic imaging was performed at the 3rd (IQR: 2-4) and 9th (IQR: 8-9) day after stroke. Patients thrombosis occurring between the first and second examination comprised the newly developed early stroke-related DVT group. We found DVT in 8.0\% (24/299) of patients at initial evaluation. Newly developed DVT was present in 3.0\% (9/299) of patients, and was predominantly distal (7 of 9 cases). It was associated with elevated serum CRP level (OR 8.75; 95\%CI: 1.61-47.6), which was verified in a model adjusted for stroke severity and pre-stroke dependency (3-5 pts. in mRS). In a multivariate model, newly developed DVT significantly increased the risk of 3-month mortality (OR 12.4; 95\%CI: 1.72-89.4), without affecting the combined risk of dependency and death (OR 2.57; 95\% CI: 0.39-17.0). Early stroke-related DVT is an infrequent complication. However, it may be an independent risk factor for 3-month mortality. Increased serum CRP
\end{abstract}

J. Bembenek $(\bowtie) \cdot$ M. Karlinski · A. Kobayashi .

A. Czlonkowska

2nd Department of Neurology, Institute of Psychiatry and Neurology, ul. Sobieskiego 9, 02-957 Warsaw, Poland e-mail: jbembenek@o2.pl

A. Czlonkowska

Department of Experimental and Clinical Pharmacology,

Medical University of Warsaw, Warsaw, Poland level combined with normal fibrinogen level seems predictive for development of DVT. It may be reasonable to provide those patients with additional DVT prophylaxis.

Keywords Deep vein thrombosis - Stroke ·

Epidemiology $\cdot$ Risk factors $\cdot$ Outcome

\section{Introduction}

Deep vein thrombosis (DVT), including pulmonary embolism (PE) as a sequel, is a serious complication of various medical conditions including stroke. It is considered to develop mostly within 2 weeks post-stroke [1]. The incidence in immobilized post-stroke patients ranges from 10 to $75 \%$, depending on the diagnostic method and time of evaluation [1-5]. According to the literature, the major risk factors of post-stroke DVT are older age [6], atrial fibrillation [7] and limb paresis [8].

Most studies addressing the issue of DVT and stroke tend to focus on patients with lower limb paresis and search for in-hospital DVT. The initial ultrasound examination is usually performed after 7 days from stroke onset, and the second one $2-5$ weeks after stroke. Such an approach is sufficient to evaluate the incidence of DVT in those patients. However, it does not allow to distinguish between early stroke-related and late onset DVT, nor with DVT probably present before. We assume this difference is of particular importance, as the late onset DTV is mainly associated with prolonged immobility, and the early onset DVT may be more likely a direct consequence of the ischaemic cerebrovascular event.

The aim of our study was to establish the occurrence of early stroke-related DVT, risk factors for its development and the influence on 3-month outcome. 


\section{Materials and methods}

We recruited consecutive patients admitted to our department due to acute stroke from December 2007 to May 2009, excluding the period from May to August 2008. The diagnosis of stroke was based on clinical symptoms and brain $\mathrm{CT}$ imaging.

Neurological deficit on admission was measured with the National Institutes of Health Stroke Scale (NIHSS). Stroke severity was categorized as mild (NIHSS $\leq 7 \mathrm{pts}$.), moderate (NIHSS 8-14 pts.) or severe (NIHSS $>14$ pts.). Pre-stroke disability was measured with Modified Rankin Scale (mRS). Information about pre-existing comorbidities and oral anticoagulation or heparins was obtained from patients medical records, patients themselves or their proxies if necessary. We also collected data on serum routine CRP level (immuno-turbidimetric method, Synchron CX 7, Beckman Coulter) and serum fibrinogen level (Claus method, Synchron CX 7, Beckman Coulter) measured within $24 \mathrm{~h}$ from hospital admission.

The follow-up examination was provided 3 months after stroke onset by a physician blinded to the patient's DVT status during a routine outpatient visit or by phone.

Primary outcome measures were overall mortality, and combined death or dependency (3-6 pts. in mRS).

\section{Ultrasonographic examination}

Patients were examined for both proximal (popliteal, femoral and common femoral vein) and distal (peroneal and tibial veins) DVT. The first ultrasonographic examination was performed within the first 7 days and then $8-10$ after stroke onset by a trained physician (JB) blinded to patients' baseline health status in order to identify patients in whom DVT occurred early in the course of stroke. We used Vivid 7 Dimension (GE, USA) with the 7-10 Hz linear probe. The diagnosis of DVT was based either on presence of a non-compressible segment (compression ultrasound test-CUS) or the flow impairment on color Doppler imaging. This method is recognized as sufficiently sensitive and specific, especially in proximal DVT detection [9].

\section{DVT prevention}

None of DVT prevention methods were used routinely. Patients at high risk of developing DVT received low molecular weight heparins (LMWH) at physican's discretion. Patients diagnosed with DVT were treated with full dose LMWH.

\section{Ethics committee approval}

The study protocol was approved by the local Ethics committee. As DVT screening with Doppler USG is noninvasive and safe, we did not collect patients written consent for the examination. The follow-up outpatient visit after 3 months is a routine element of post stroke care in our department.

\section{Statistical methods}

Categorical variables were presented as ratio with number of valid observations, and continuous variables as median with interquartile range (IQR). Proportions were calculated with exclusion of unknown values from the denominator. In basic comparative statistics we applied chi square test or two sided exact Fisher's test, and Mann-Whitney $U$ test, for categorical and continuous variables, respectively.

Multivariate logistic regression was adjusted for all independent outcome predictors. To avoid variable selection caused by spurious correlations, only variables showing a relationship to the outcome (defined $P<0.10$ in the univariate model) were included as potential predictors. We identify the final multivariate model for each major outcome using a backward stepwise approach with the $P<0.05$ of the likelihood ratio test for exclusion of excess factors.

We considered $P$ value $<0.05$ statistically significant. Analyses were conducted in STATISTICA 8.0 (StatSoft, Inc. 2008).

\section{Results}

During the recruitment period of 16 months a total of 425 acute stroke patients were admitted to our stroke unit. We excluded 102 patients who were not able to undergo initial USG evaluation due to early transfer to another hospital, death or unstable general condition not allowing to transfer the patients to the USG laboratory. We also excluded 24 patients who were lost from the follow-up USG due to reasons similar as mentioned above.

Patients excluded from the study had more severe neurological deficit at baseline, and more frequently decreased level of consciousness. 39 (31\%) of them died during the hospital stay at a median of 2 days (IQR: 2-4). A detailed description of both groups is presented in Table 1 .

The final analysis involved 299 Caucasian patients with acute ischaemic (92.8\%) and haemorrhagic (7.2\%) stroke. The initial and follow-up USG examinations were performed at the 3rd (IQR: 2-4) and 9th (IQR: 8-9) day after stroke, respectively. The median gap between examinations was 6 (IQR: 4-7) days. 
Table 1 Comparison of the study group and patients not included in the study

\begin{tabular}{|c|c|c|c|c|c|}
\hline & \multicolumn{2}{|c|}{ Not included } & \multicolumn{2}{|c|}{ Included } & \multirow[t]{2}{*}{$P$} \\
\hline & $\mathrm{N}$ & Observed $(\%)$ & $\mathrm{N}$ & Observed $(\%)$ & \\
\hline Age (median, IQR) & 126 & $72(62-81)$ & 299 & $75(64-82)$ & 0.162 \\
\hline Female sex & 126 & $62(49.2 \%)$ & 299 & $159(53.2 \%)$ & 0.454 \\
\hline Arterial hypertension & 126 & $89(70.6 \%)$ & 288 & $214(74.3 \%)$ & 0.438 \\
\hline Congestive heart failure & 123 & $32(26.0 \%)$ & 290 & $72(24.8 \%)$ & 0.799 \\
\hline Atrial fibrillation & 123 & $30(24.4 \%)$ & 290 & $61(21.0 \%)$ & 0.452 \\
\hline Diabetes & 124 & $16(12.9 \%)$ & 297 & $54(18.2 \%)$ & 0.185 \\
\hline \multicolumn{6}{|l|}{ Smoking status } \\
\hline Current smokers & 123 & $28(22.8 \%)$ & 298 & $74(24.8 \%)$ & 0.652 \\
\hline Past smokers & 122 & $55(45.1 \%)$ & 296 & $140(47.3 \%)$ & 0.680 \\
\hline Oral anticoagulants & 125 & $14(11.2 \%)$ & 296 & $21(7.1 \%)$ & 0.163 \\
\hline \multicolumn{6}{|l|}{ Pre-stroke disability } \\
\hline mRS $0-1$ pt. & 126 & $85(67.5 \%)$ & 299 & $218(72.9 \%)$ & 0.257 \\
\hline mRS $0-2$ pts. & 126 & $95(75.4 \%)$ & 299 & $246(82.3 \%)$ & 0.104 \\
\hline Stroke severity (median, IQR\%) & 126 & $8(3-20)$ & 299 & $5(2-9)$ & $<0.001$ \\
\hline NIHSS $\leq 7$ pts. & 126 & $59(46.8 \%)$ & 299 & $205(68.6 \%)$ & $<0.001$ \\
\hline NIHSS $8-14$ pts. & 126 & $20(15.9 \%)$ & 299 & $52(17.4 \%)$ & 0.703 \\
\hline NIHSS $>14$ pts. & 126 & $47(37.3 \%)$ & 299 & $42(14.0 \%)$ & $<0.001$ \\
\hline \multicolumn{6}{|l|}{ Decreased consciousness } \\
\hline Not present & 126 & $78(61.9 \%)$ & 299 & $256(85.6 \%)$ & $<0.001$ \\
\hline 1 pt. in NIHSS & 126 & $24(19.0 \%)$ & 299 & $30(10.0 \%)$ & 0.011 \\
\hline$\geq 2$ pts. in NIHSS & 126 & $24(19.0 \%)$ & 299 & $13(4.3 \%)$ & $<0.001$ \\
\hline
\end{tabular}

We found DVT in $24(8.0 \%)$ patients at initial and in 32 (10.7\%) patients at follow-up examination. In one case, the clot resolved before the second examination. Therefore, newly developed DVT was present in nine (3.0\%) patients, and was predominantly distal (7 cases).

Patients with newly developed DVT despite a trend for younger age (median 68 vs. $75 ; P=0.429$ ) tended to have higher ratio of pre-existing diabetes (33.3 vs. 17.7\%; $P=0.213)$ and congestive heart failure (44.4 vs. $24.2 \%$; $P=0.233)$. There was also a trend for higher stroke severity (median NIHSS score 8 vs. 5; $P=0.090$ ), including more frequently decreased consciousness (33.3 vs. $13.8 \% ; P=0.125)$. Patients with DVT had significantly higher ratio of elevated serum CRP level (77.8 vs. $32.6 \% ; P=0.008)$ with a strong inverse trend for elevated serum fibrinogen level (44.4 vs. $73.6 \% ; P=0.067$ ). Detailed characteristic of the study population is presented in Table 2.

In a multivariate model, development of early strokerelated DVT was associated with elevated serum CRP concentration and not-increased serum fibrinogen concentration. There was also a positive trend for pre-stroke diabetes. Pre-stroke dependency and stroke severity did not show significant association, nor trend for increased risk of DVT. Detailed OR for early stroke-related DVT are presented in Table 3.
Patients with early stroke-related DVT had significantly higher 3-month mortality (42.9 vs. 9.2\%, $P=0.03$ ), and slightly more frequently developed death or dependency (57.1 vs. $41.1 \%, P=0.45$ ). In multivariate logistic regression early stroke-related DVT was associated with increased risk of death at 3 months (OR 12.4; 95\%CI: 1.72-89.4), after adjusting for the independent predictors (i.e. older age, use of oral anticoagulants, stroke severity and elevated CRP concentration) (Table 4).

Early stroke-related DVT did not significantly influence 3-month death or dependency. After adjusting for the independent predictors (i.e. stroke severity and pre-stroke disability level), the OR in early stroke-related DVT was 2.57 (95\%CI: 0.39-17.0). It also did not influence the outcome in survivors. After adjusting for the independent predictors (i.e. age, stroke severity and pre-stroke disability level), the OR for dependency in survivors (mRS 2-5 pts.) in early stroke-related DVT patients was 1.83 (95\% CI: 0.17-20.1) (Table 4).

\section{Discussion}

Our findings show, that the frequency of DVT in acute stroke patients reaches $10.7 \%$. However, in $8 \%$ of cases is may have developed before the stroke, while only in $3 \%$ 
Table 2 Baseline characteristics of the study population and 3-month outcomes

\begin{tabular}{|c|c|c|c|c|c|}
\hline & \multicolumn{2}{|c|}{$\begin{array}{l}\text { Newly developed DVT } \\
\text { group }\end{array}$} & \multicolumn{2}{|c|}{$\begin{array}{l}\text { Old DVT or non-DVT } \\
\text { group }\end{array}$} & \multirow[t]{2}{*}{$P$} \\
\hline & $\mathrm{N}$ & Observed $(\%)$ & $\mathrm{N}$ & Observed (\%) & \\
\hline Age (median, IQR) & 9 & $68.0(63-79)$ & 290 & $75(65-82)$ & 0.429 \\
\hline Female sex & 9 & $5(55.6 \%)$ & 290 & $154(53.1 \%)$ & 1.000 \\
\hline Arterial hypertension & 9 & $7(77.8 \%)$ & 279 & $207(74.2 \%)$ & 1.000 \\
\hline Congestive heart failure & 9 & $4(44.4 \%)$ & 281 & $68(24.2 \%)$ & 0.233 \\
\hline Atrial fibrillation & 9 & $2(22.2 \%)$ & 281 & $59(21.0 \%)$ & 1.000 \\
\hline Diabetes & 9 & $3(33.3 \%)$ & 288 & $51(17.7 \%)$ & 0.213 \\
\hline \multicolumn{6}{|l|}{ Smoking status } \\
\hline Current smokers & 9 & $2(22.2 \%)$ & 289 & $72(24.9 \%)$ & 1.000 \\
\hline Past smokers & 9 & $4(44.4 \%)$ & 287 & $136(47.4 \%)$ & 1.000 \\
\hline Oral anticoagulants & 9 & $0(0 \%)$ & 287 & $21(7.3 \%)$ & 1.000 \\
\hline \multicolumn{6}{|l|}{ Pre-stroke disability } \\
\hline mRS $0-1$ pt. & 9 & $6(66.7 \%)$ & 290 & $222(74.1 \%)$ & 0.707 \\
\hline mRS $0-2$ pts. & 9 & $8(88.9 \%)$ & 290 & $238(82.1 \%)$ & 1.000 \\
\hline Ischaemic stroke & 9 & $9(100.0 \%)$ & 290 & $269(92.8 \%)$ & 1.000 \\
\hline Stroke severity [median, IQR] & 9 & $8(4-20.5)$ & 290 & $5(2-9)$ & 0.090 \\
\hline NIHSS $\leq 7$ pts. & 9 & $4(44.4 \%)$ & 290 & $201(69.3 \%)$ & 0.146 \\
\hline NIHSS $8-14$ pts. & 9 & $3(33.3 \%)$ & 290 & $49(16.9 \%)$ & 0.193 \\
\hline NIHSS $>14$ pts. & 9 & $2(22.2 \%)$ & 290 & $40(138 \%)$ & 0.368 \\
\hline \multicolumn{6}{|l|}{ Decreased consciousness } \\
\hline Not present & 9 & $6(66.7 \%)$ & 290 & $250(86.2 \%)$ & 0.125 \\
\hline 1 pt. in NIHSS & 9 & $2(22.2 \%)$ & 290 & $28(9.7 \%)$ & 0.225 \\
\hline$\geq 2$ pts. in NIHSS & 9 & $1(11.1 \%)$ & 290 & $12(4.1 \%)$ & 0.333 \\
\hline \multicolumn{6}{|l|}{ Inflammatory markers } \\
\hline $\mathrm{CRP}>10 \mathrm{mg} / \mathrm{dl}$ & 9 & $7(77.8 \%)$ & 276 & $90(32.6 \%)$ & 0.008 \\
\hline Fibrinogen $>4 \mathrm{mg} / \mathrm{dl}$ & 9 & $4(44.4 \%)$ & 276 & $203(73.6 \%)$ & 0.067 \\
\hline Fibrinogen [median, IQR] & 9 & $3.8(3.4-5.8)$ & 276 & $4.7(4.0-5.8)$ & 0.230 \\
\hline \multicolumn{6}{|l|}{ Stroke outcome at 3 months } \\
\hline Mortality & 7 & $3(42.9 \%)$ & 275 & $27(9.8 \%)$ & 0.028 \\
\hline Death or dependency (mRS 3-6\%) & 7 & $4(57.1 \%)$ & 275 & $113(41.1 \%)$ & 0.454 \\
\hline Dependency in survivors (mRS $3-5 \%$ ) & 4 & $1(25.0 \%)$ & 248 & $86(34.7 \%)$ & 1.000 \\
\hline
\end{tabular}

between the 3rd and 9th day after stroke. According to previous studies, the incidence of DVT within the first 14 days post-stroke ranges from 10 to $75 \%$ of patients, depending on the applied methodology [1-5].

In a recent large trial by Dennis et al. [10] a total of 2518 acute stroke patients randomized to thigh-length graduated compression stockings for DVT prevention $(n=1256)$ or routine care ( $n=1262$ ) were evaluated with a compression ultrasound test at 7-10 days after stroke and if possible at 25-30 days after enrollment. In the initial examination, the ratio of combined symptomatic and asymptomatic DVT was $10.0 \%$ in the study group and $10.5 \%$ in the control group, which is fully consistent with our results. In another USG-based study, De Silva et al. assessed the incidence of DVT in 105 acute ischaemic stroke Asian patients. At the first evaluation performed 7-10 days after stroke they found DVT in 30\% of patients. On follow-up evaluation, performed 25-30 days after stroke, DVT was detected in $45 \%$ of patients. Those results may suggest that Asian population is more prone to develop DVT, when compared to Caucasians [11]. The protocol used in our study differed from previous ones and included two USG examinations performed in the acute phase of stroke within a gap of a few days. This allowed us to differentiate patients in whom DVT was most likely a direct consequence of the acute stroke, and not chronic immobility or pre-stroke thrombosis.

In our study, newly developed early stroke-related DVT was predominantly distal ( 7 of 9 cases), which stays in concordance with other studies [5, 11-14]. We decided to search for distal DVT as it also poses an indirect but significant threat, as the thrombi propagates above the knee in 
Table 3 Unadjusted and adjusted odds ratio for early stroke-related DVT

\begin{tabular}{|c|c|c|c|c|c|c|}
\hline & \multicolumn{3}{|c|}{ Univariate model } & \multicolumn{3}{|c|}{ Multivariate model* } \\
\hline & OR & $95 \% \mathrm{CI}$ & $P$ & OR & $95 \% \mathrm{CI}$ & $P$ \\
\hline Age (for each additional 10 years) & 0.91 & $(0.70-1.19)$ & 0.507 & 0.64 & $(0.33-1.23)$ & 0.176 \\
\hline Female sex & 1.10 & $(0.29-4.22)$ & 0.885 & 0.81 & $(0.20-3.37)$ & 0.775 \\
\hline Hypertension & 1.22 & $(0.25-6.04)$ & 0.809 & 1.44 & $(0.27-7.61)$ & 0.665 \\
\hline Congestive heart failure & 2.51 & $(0.65-9.65)$ & 0.180 & 1.71 & $(0.41-7.05)$ & 0.458 \\
\hline Atrial fibrillation & 1.08 & $(0.22-5.34)$ & 0.929 & 0.65 & $(0.12-3.48)$ & 0.609 \\
\hline Diabetes & 2.32 & $(0.56-9.65)$ & 0.244 & 4.16 & $(0.84-20.6)$ & 0.079 \\
\hline \multicolumn{7}{|l|}{ Smoking status } \\
\hline Current smoker & 0.86 & $(0.17-4.37)$ & 0.854 & 1.59 & $(0.28-8.89)$ & 0.599 \\
\hline Previous smoker & 0.89 & $(0.23-3.39)$ & 0.862 & 0.97 & $(0.24-3.93)$ & 0.969 \\
\hline Pre-stroke disability & 1.05 & $(0.65-1.72)$ & 0.834 & 0.97 & $(0.58-1.62)$ & 0.923 \\
\hline mRS $0-1$ pt. & 0.74 & $(0.18-3.03)$ & 0.670 & 0.95 & $(0.21-4.23)$ & 0.947 \\
\hline mRS $0-2$ pts. & 1.75 & $(0.21-14.4)$ & 0.602 & 0.44 & $(0.05-3.90)$ & 0.460 \\
\hline \multicolumn{7}{|l|}{ Stroke severity } \\
\hline Each additional 4 pts. NIHSS & 1.30 & $(0.96-1.75)$ & 0.083 & 1.21 & $(0.86-1.69)$ & 0.271 \\
\hline NIHSS $>7$ pts. & 2.82 & $(0.74-10.8)$ & 0.129 & 2.11 & $(0.50-8.96)$ & 0.307 \\
\hline NIHSS $>14$ pts. & 1.79 & $(0.35-8.96)$ & 0.479 & 1.34 & $(0.25-7.30)$ & 0.732 \\
\hline \multicolumn{7}{|l|}{ Decreased consciousness } \\
\hline$\geq 1$ pt. in NIHSS & 3.13 & $(0.75-13.1)$ & 0.117 & 2.09 & $(0.46-9.54)$ & 0.337 \\
\hline$\geq 2$ pts. in NIHSS & 2.90 & $(0.33-25.3)$ & 0.334 & 1.90 & $(0.19-19.0)$ & 0.583 \\
\hline \multicolumn{7}{|l|}{ Inflammatory markers } \\
\hline $\mathrm{CRP}>10 \mathrm{mg} / \mathrm{l}$ & 7.23 & $(1.46-35.8)$ & 0.015 & 10.1 & $(1.93-52.9)$ & 0.006 \\
\hline Fibrinogen $>4 \mathrm{mg} / \mathrm{dl}$ & 0.29 & $(0.07-1.11)$ & 0.069 & 0.18 & $(0.04-0.74)$ & 0.017 \\
\hline
\end{tabular}

* Adjusted for elevated blood CRP level (>10 mg/dl) and fibrinogen level (>4 mg/dl)

Table 4 Adjusted odds ratio for 3-month mortality and unfavorable outcome (mRS 2-6)

\begin{tabular}{|c|c|c|c|c|c|c|}
\hline & \multicolumn{3}{|c|}{ Death at 3 months* } & \multicolumn{3}{|c|}{ Death or dependency ${ }^{\mathrm{a}}$} \\
\hline & OR & $95 \% \mathrm{CI}$ & $P$ & OR & $95 \% \mathrm{CI}$ & $P$ \\
\hline Newly developed DVT & 12.4 & $(1.72-89.4)$ & 0.012 & 2.57 & $(0.39-17.0)$ & 0.324 \\
\hline Age (for each additional 10 years) & 2.05 & $(1.17-3.59)$ & 0.011 & 1.55 & $(1.14-2.10)$ & 0.005 \\
\hline Oral anticoagulants & 5.58 & $(1.11-28.1)$ & 0.036 & & & \\
\hline Pre-stroke nondisability (mRS $0-1$ ) & & & & 0.25 & $(0.12-0.50)$ & $<0.001$ \\
\hline Stroke severity (for each 4 NIHSS) & 1.78 & $(1.36-2.31)$ & $<0.001$ & 2.79 & $(2.08-3.74)$ & $<0.001$ \\
\hline $\mathrm{CRP}>10 \mathrm{mg} / \mathrm{l}$ & 2.94 & $(1.08-7.96)$ & 0.034 & & & \\
\hline
\end{tabular}

* Model adjusted for all presented variables; ${ }^{\text {a }}$ Model adjusted for all presented excluding newly developed DVT

even up to $20 \%$ of cases $[15,16]$. Therefore, the risk of proximal extension and possibility of developing PE as a sequel should not be neglected [17].

We assume that the cases of newly developed DVT may be significantly different from those diagnosed in other studies using only one USG examination during the first 2 weeks post stroke. In our study population, patients presenting with DVT at the first USG examination were less independent before stroke and did not show any increase in 3-month mortality [18]. Therefore, we cannot be certain when the clot formation was triggered. It is possible, that in many cases DVT was already present before stroke onset.

The results of other studies provide evidence that the development of DVT is associated with elevation of systemic inflammatory markers $[19,20]$, which is partially consistent with our findings.

However, the issue of cause-and-results relationship of increased inflammatory markers and DVT is still a matter of debate. Besides, the number of published studies on acute stroke patients is very limited. In this aspect, our study provides a new perspective. It allows us to 
distinguish patients developing stroke-related DVT in a very narrow time window after baseline evaluation, as the ultrasound examinations were performed with the median interval of 4 days. Such design gives information about the level of inflammatory markers directly preceding the clot formation, and has not been applied before. In other studies the gap between USG evaluation usually ranges from 2 to 4 weeks $[10,11]$.

The increase of CRP level in patients with DVT, that we observed in our study, is fully consistent with published literature. However other studies, have not concentrated on stroke patients [19, 21, 22].

Surprisingly, our findings show that early stroke related DVT is inversely associated with elevated fibrinogen level. Unfortunately, available studies supporting the positive association between DVT and serum fibrinogen were conducted on small groups of patients, did not have such a narrow time window and did not address directly the acute stroke [23-27]. Therefore, it is difficult to make direct comparisons. We may speculate, that observed in our study inverse association between the elevated fibrinogen level and the development of DVT is a result of fibrinogen depletion due to active clot formation. However, this assumption is based on a small group of stroke patients, and gives a good rationale for further investigations.

In a study by Wang et al. the mean levels of plasma CRP and fibrinogen were significantly higher in 59 DVT patients compared to 26 healthy controls, i.e. $2.67 \pm 0.91$ versus $0.14 \pm 0.08 \mathrm{mg} / \mathrm{dl}$. Similar observation was made for fibrinogen ( $4.73 \pm 1.36$ vs. $2.79 \pm 0.66 \mathrm{~g} / \mathrm{l}$, respectively). The authors suggest that interaction between inflammation and coagulation promote the development of DVT and may be involved in DVT pathogenesis [23]. Unfortunately, this was a small study and only the abstract is available in English. Therefore, its methodology stays unclear.

Ogata et al. [28] attempted to detect DVT in 56 acute haemorrhagic stroke patients within $72 \mathrm{~h}$ from the onset of symptoms and after 2 weeks using ultrasonography. They did not find any significant correlation between fibrinogen level and risk of DVT. However, trials not concentrating directly on stroke patients suggest that elevated fibrinogen level is associated with even a 4-fold increase in the risk of developing DVT [24-27].

According to our findings, pre-stroke disability level and stroke severity were independent predictors of achieving unfavorable outcome during the follow-up, which stays in concordance with other studies [29-32].

It is generally agreed that proper DVT prophylaxis improves stroke outcome, although there is no evidence supporting this thesis [33]. In our study early stroke-related DVT was an independent risk factor for death at 3 months with a slight trend for unfavorable outcome in survivors. De Silva et al. [11] reported that the presence of DVT
25-30 days after stroke was associated with higher ratio of poor outcome (defined as mRS 4-6) after 6 months (50 vs. $26 \% ; P=0.024)$. In patients diagnosed with DVT during 7-10 days after stroke this association was not statistically significant (52 vs. $32 \% ; P=0.074$ ). Therefore, patients with acute stroke should be carefully screened for DVT risk factors at admission. Throughout the hospitalization they should also be at least clinically monitored for the development of DVT. It applies to all patients regardless of stroke severity, especially if serum CRP levels are elevated.

None of our patients was diagnosed with PE, however, the majority of deaths occurred after discharge from the hospital. We may speculate, that early stroke-related DVT is a clinical marker of other discrete conditions that increase the risk of death. It is also possible that those patients are more likely to have serious clinical course of thrombosis with higher risk of PE despite applied treatment.

Our study has certain limitations. Although ultrasound is highly sensitive and specific in detection of proximal DVT in symptomatic patients, it's sensitivity for distal DVT detection is much lower than proximal (62.1 vs. 93.9\%) [34]. The sample size of early stroke-related DVT was too small (9 cases) to draw strong conclusions. Our cohort is skewed towards relatively stable patients with mild-tosevere strokes not requiring early transfer to other specialist units. Only two basic inflammation markers were tested in this study. We also did not perform routine autopsy in patients who died during the study period, therefore we cannot exclude that some deaths were due to PE.

In conclusion, our study shows that DVT which is definitely associated with acute stroke occurs in $3 \%$ of patients and significantly affects 3-month mortality. Elevated serum CRP level and not-increased fibrinogen serum level are independently associated with increased risk of DVT. Therefore, it may be reasonable to provide this group of patients with additional care and proper DVT prophylaxis in order to minimize the risk of thrombo-embolic complications.

Open Access This article is distributed under the terms of the Creative Commons Attribution Noncommercial License which permits any noncommercial use, distribution, and reproduction in any medium, provided the original author(s) and source are credited.

\section{References}

1. Harvey RL (2003) Prevention of venous thromboembolism after stroke. Top Stroke Rehabil 10(3):61-69

2. Warlow C, Ogston D, Douglas AS (1976) Deep venous thrombosis of the legs after strokes. Part 1. Incidence and predisposing factors. BMJ 1:1178-1181

3. Cope C, Tyrone MR, Skversky NJ (1973) Phlebographic analysis of the incidence of thrombosis in hemiplegia. Radiology 109:581-584 
4. Oczkowski WJ, Ginsberg JS, Shin A, Panju A (1992) Venous thromboembolism in patients undergoing rehabilitation for stroke. Arch Phys Med Rehabil 73:712-716

5. Kelly J, Rudd A, Lewis RR, Coshall C, Moody A, Hunt BJ (2004) Venous thromboembolism after acute ischemic stroke: a prospective study using magnetic resonance direct thrombus imaging. Stroke 35(10):2320-2325

6. Mulley GP (1982) Avoidable complications of stroke. J R Coll Physicians Lond 16(2):94-97

7. Noel P, Gregoire F, Capon A, Lehert P (1991) Atrial fibrillation as a risk factor for deep venous thrombosis and pulmonary emboli in stroke patients. Stroke 22(6):760-762

8. Landi G, D'Angelo A, Boccardi E, Candelise L, Mannucci PM, Morabito A, Orazio EN (1992) Venous thromboembolism in acute stroke. Prognostic importance of hypercoagulability. Arch Neurol 49(3):279-283

9. Tapson VF, Carroll BA, Davidson BL, Elliott CG, Fedullo PF, Hales CA, Hull RD, Hyers TM, Leeper KV Jr, Morris TA, Moser KM, Raskob GE, Shure D, Sostman HD, Taylor Thompson B (1999) The diagnostic approach to acute venous thromboembolism. Clinical practice guideline. American Thoracic Society. Am J Respir Crit Care Med 160(3):1043-1066

10. CLOTS Trials Collaboration, Dennis M, Sandercock PA, Reid J, Graham C, Murray G, Venables G, Rudd A, Bowler G (2009) Effectiveness of thigh-length graduated compression stockings to reduce the risk of deep vein thrombosis after strokenext term (CLOTS trial 1): a multicentre, randomised controlled trial. Lancet 373(9679):1958-1965

11. De Silva DA, Pey HB, Wong MC, Chang HM, Chen CP (2006) Deep vein thrombosis following ischemic stroke among Asians. Cerebrovasc Dis 22(4):245-250

12. Kearon C (2003) Natural history of venous thromboembolism. Circulation 107:122-130

13. Lacut K, Bressollette L, Le Gal G, Etienne E, De Tinteniac A, Renault A, Rouhart F, Besson G, Garcia JF, Mottier D, Oger E on behalf of the VICTORIAh (Venous Intermittent Compression and Thrombosis Occurrence Related to Intra-cerebral Acute hemorrhage) investigators (2005) Prevention of venous thrombosis in patients with acute intracerebral hemorrhage. Neurology 65(6): 865-869

14. Kong KH, Chua SG, Earnest A (2009) Deep vein thrombosis in stroke patients admitted to a rehabilitation unit in Singapore. Int J Stroke 4(3): 175-179

15. Ascher E, Depippo PS, Hingorani A, Yorkovich W, Salles-Cunha S (2004) Does repeat duplex ultrasound for lower extremity deep vein thrombosis influence patient management? Vasc Endovascular Surg 38(6):525-531

16. Philbrick JT, Becker DM (1988) Calf deep venous thrombosis. A wolf in sheep's clothing? Arch Intern Med 148(10):2131-2138

17. Kelly J, Rudd A, Lewis R, Hunt BJ (2001) Venous thromboembolism after acute stroke. Stroke 32(1):262-267

18. Bembenek J, Karlinski M, Kobayashi A, Czlonkowska A (2010) Deep venous thrombosis in acute stroke patients. Cerebrovascular Diseases 29 (suppl 2)

19. Reiter M, Bucek RA, Koca N, Dirisamer A, Minar E (2003) Deep vein thrombosis and systemic inflammatory response: a pilot trial. Wien Klin Wochenschr 115(3-4):111-114
20. Bucek RA, Reiter M, Quehenberger P, Minar E (2002) C-reactive protein in the diagnosis of deep vein thrombosis. Br J Haematol 119(2):385-389

21. Fox EA, Kahn SR (2005) The relationship between inflammation and venous thrombosis. A systematic review of clinical studies. Thromb Haemost 94(2):362-365

22. Ozbudak O, Eroğullari I, Oğüş C, Cilli A, Türkay M, Ozdemir T (2006) Doppler ultrasonography versus venography in the detection of deep vein thrombosis in patients with pulmonary embolism. J Thromb Thrombolysis 21(2):159-162

23. Wang MF, Yang LH, Yang XL, Zhang RJ, Hou LH, Liu XE (2010) Correlation of inflammatory marker and coagulation factors with deep vein thrombosis. Zhongguo Shi Yan Xue Ye Xue Za Zhi 18(3):753-756

24. Salobir B, Sabovic M, Peternel P, Stegnar M (2003) Vascular bed specific alterations in coagulation and fibrinolytic parameters in young women following myocardial infarction, lacunar cerebral infarction and deep vein thrombosis. Pathophysiol Haemost Thromb 33(2):96-101

25. Austin H, Hooper WC, Lally C, Dilley A, Ellingsen D, Wideman C, Wenger NK, Rawlins P, Silva V, Evatt B (2000) Venous thrombosis in relation to fibrinogen and factor VII genes among African-Americans. J Clin Epidemiol 53(10):997-1001

26. Kamphuisen PW, Eikenboom JC, Vos HL, Pablo R, Sturk A, Bertina RM, Rosendaal FR (1999) Increased levels of factor VIII and fibrinogen in patients with venous thrombosis are not caused by acute phase reactions. Thromb Haemost 81(5):680-683

27. Koster T, Rosendaal FR, Reitsma PH, van der Velden PA, Briët E, Vandenbroucke JP (1994) Factor VII and fibrinogen levels as risk factors for venous thrombosis-A case-control study of plasma levels and DNA polymorphisms-The Leiden thrombophilia study (LETS). Thromb Haemost 71:719-722

28. Ogata T, Yasaka M, Wakugawa Y, Inoue T, Ibayashi S, Okada Y (2008) Deep venous thrombosis after acute intracerebral hemorrhage. J Neurol Sci 272(1-2):83-86

29. Bassi A, Colivicchi F, Santini M, Caltagirone C (2010) Genderspecific predictors of functional outcome after stroke rehabilitation: potential role of the autonomic nervous system. Eur Neurol 63(5):279-284

30. Denti L, Scoditti U, Tonelli C, Saccavini M, Caminiti C, Valcavi R, Benatti M, Ceda GP (2010) The poor outcome of ischemic stroke in very old people: a cohort study of its determinants. J Am Geriatr Soc 58(1):12-17

31. Appelros P, Nydevik I, Viitanen M (2003) Poor outcome after first-ever stroke: predictors for death, dependency, and recurrent stroke within the first year. Stroke 34(1):122-126

32. Christensen H, Boysen G (2004) C-reactive protein and white blood cell count increases in the first $24 \mathrm{~h}$ after acute stroke. Cerebrovasc Dis 18:214-219

33. Bravata DM, Wells CK, Lo AC, Nadeau SE, Melillo J, Chodkowski D, Struve F, Williams LS, Peixoto AJ, Gorman M, Goel P, Acompora G, McClain V, Ranjbar N, Tabereaux PB, Boice JL, Jacewicz M, Concato J (2010) Processes of care associated with acute stroke outcomes. Arch Intern Med 170(9):804-810

34. Sampson FC, Goodacre SW, Thomas SM, van Beek EJ (2007) The accuracy of MRI in diagnosis of suspected deep vein thrombosis: systematic review and meta-analysis. Eur Radiol 17(1):175-181 